\title{
Equilibrium and Structural Studies of Silicon(IV) and Aluminium(III) in Aqueous Solution. 30. Aqueous Complexation between Silicic Acid and Some ortho-Di- and Triphenolic Compounds
}

\author{
Iran Fattahpour Sedeh, Lars-Olof Öhman* and Staffan Sjöberg \\ Department of Inorganic Chemistry, University of Umeå, S-901 87 Umeå, Sweden
}

\begin{abstract}
Fattahpour Sedeh, I., Öhman, L.-O. and Sjöberg, S., 1992. Equilibrium and Structural Studies of Silicon(IV) and Aluminium(III) in Aqueous Solution. 30. Aqueous Complexation between Silicic Acid and Some ortho-Di- and Triphenolic Compounds.

- Acta Chem. Scand. 46: 933-940.

Three-component equilibria between $\mathrm{H}^{+}$, silicic acid and the three compounds pyrogallol, protocatechuic acid and gallic acid, all with phenolic groups in the ortho position, have been studied by means of EMF titrations at $25^{\circ} \mathrm{C}$ in an ionic medium of $0.6 \mathrm{M} \mathrm{Na}(\mathrm{Cl})$. In all three systems, and in accordance with a previous investigation of $\mathrm{Si}(\mathrm{OH})_{4}$ complexation to pyrocatechol, data can be explained with a single, mononuclear, tris-complex $\mathrm{SiL}_{3}$. The charge of the complex is -2 for pyrogallol and -5 for protocatechuic acid and gallic acid.

Data were analyzed with the least-squares computer program LETAGROPVRID. The equilibrium constants with corresponding standard deviations were determined using data from slightly basic solutions $\left(6.5 \leq-\log \left[\mathrm{H}^{+}\right] \leq 9.5\right)$. For pyrogallol, the equilibrium constant is $\log K\left[\mathrm{Si}(\mathrm{OH})_{4}+3 \mathrm{H}_{3} \mathrm{~L} \rightleftarrows \mathrm{Si}(\mathrm{HL})_{3}{ }^{2-}+2 \mathrm{H}^{+}+4 \mathrm{H}_{2} \mathrm{O}\right]$ $-10.02 \pm 0.015$. Corresponding constants for protocatechuic acid and gallic acid are $\log K\left[\mathrm{Si}(\mathrm{OH})_{4}+3 \mathrm{H}_{3} \mathrm{~L} \rightleftarrows \mathrm{SiL}_{3}^{5-}+5 \mathrm{H}^{+}+4 \mathrm{H}_{2} \mathrm{O}\right]-21.95 \pm 0.049$ and $\log K\left[\mathrm{Si}(\mathrm{OH})_{4}+3 \mathrm{H}_{4} \mathrm{~L} \rightleftarrows \mathrm{Si}(\mathrm{HL})_{3}{ }^{5-}+5 \mathrm{H}^{+}+4 \mathrm{H}_{2} \mathrm{O}\right]-21.26 \pm 0.031$, respectively.

The octahedral oxygen coordination around the silicon atom in these complexes has been strongly indicated by using the ${ }^{29} \mathrm{Si}-\mathrm{NMR}$ method. By using pyrocatechol as a reference point, the log $K$-values for the first phenolic dissociation constant as well as the $\log K$-values for the formation of $\mathrm{SiL}_{3}$ complexes were found to be linearly correlated to the Hammett $\sigma$-constant of the ligand.
\end{abstract}

In a previous paper of this series, ${ }^{1}$ the ability of different organic and inorganic substances to raise the solubility of amorphous silica was studied. Through these measurements it was found that, in the presence of the $o$-diphenolic compound pyrocatechol and at slightly alkaline $\mathrm{pH}$, a substantial solubilization occurred. Precise EMF measurements, performed in homogeneous $\mathrm{Si}(\mathrm{OH})_{4}$-pyrocatechol solutions, showed the formation of a single, octahedrally coordinated species $\mathrm{SiL}_{3}{ }^{2-}$, and, by applying the stability of this species to the silica-catechol suspensions, a full explanation of the recorded solubilization effects was obtained.

In the present investigation, the aqueous interactions between silicic acid and three substituted pyrocatechols (protocatechuic acid, pyrogallol and gallic acid, with structures as given in Scheme 1) have been studied. The aims of the study are to evaluate whether or not the formation of a single octahedrally coordinated $\mathrm{SiL}_{3}$ species is a general feature for the interaction between $\mathrm{Si}(\mathrm{OH})_{4}$ and aromatic compounds containing two adjacent phenolic groups and, if so, whether it is possible to correlate the stability of the

* To whom correspondence should be addressed.
Gallic acid<smiles>O=C(O)c1cc(O)c(O)c(O)c1</smiles><smiles>O=C(O)c1ccc(O)c(O)c1</smiles><smiles>[X]c1cc([X])c(O)c(O)c1</smiles>

Protocatechuic acid<smiles>Oc1ccccc1</smiles>

Pyrocatechol

Scheme 1.<smiles>Oc1cccc(O)c1O</smiles>

Pyrogallol 
complex to the substituent pattern on the pyrocatechol molecule.

As in the previous study, the interpretation of data will be based on precise potentiometric measurements at low silicic acid concentrations, so that the polynuclear hydrolysis of silicic acid can be accounted for. Data will cover as wide $\mathrm{Si}(\mathrm{OH})_{4}$ /ligand ranges as possible, so that the formation of possible binary as well as ternary complexes can be examined.

\section{Experimental}

Chemicals and analysis. Aqueous solutions of sodium chloride (Merck p.a.), hydrochloric acid (Merck p.a.), sodium hydroxide (EKA p.a.) and silicic acid (Baker p.a.) were prepared as described in Ref. 1.

For the NMR measurements, ${ }^{29} \mathrm{Si}$-enriched $\mathrm{SiO}_{2}(4.7 \%$ ${ }^{28} \mathrm{Si}, 95.0 \%{ }^{29} \mathrm{Si}, 0.3 \%{ }^{30} \mathrm{Si}$, Oak Ridge National Laboratory) was used. A special procedure to prepare a carbonate-free silicate solution from this material is described in Ref. 2.

Gallic acid (3,4,5-trihydroxybenzoic acid, $\mathrm{C}_{7} \mathrm{H}_{6} \mathrm{O}_{5} \cdot \mathrm{H}_{2} \mathrm{O}$, Baker grade) was used without further purification, as every attempt to recrystallize it from hot water resulted in a pale grey, partly oxidized product.

Stock solutions of gallic acid were prepared by dissolving $\mathrm{C}_{7} \mathrm{H}_{6} \mathrm{O}_{5} \cdot \mathrm{H}_{2} \mathrm{O}$, together with the appropriate amount of $\mathrm{NaCl}$, in distilled water and standardized $\mathrm{HCl}$. The $\mathrm{C}_{7} \mathrm{H}_{6} \mathrm{O}_{5}$ content was determined potentiometrically using the Gran extrapolation method. ${ }^{3}$

The titrated amount was always higher than that expected from the weighed amount $(\approx 1.5 \%)$ owing to a water content of less than unity in the solid phase. The value obtained from titration has been assumed to be correct. After standardization, the solution was used within a few days in order to avoid effects from the slow oxidation which occurs in acidic solution.

Acidified pyrogallol (1,2,3-trihydroxybenzene, Merck p.a.) solutions were prepared and analyzed as described for the gallic acid solution. The titrated amount of $\mathrm{C}_{6} \mathrm{H}_{3}(\mathrm{OH})_{3}$ was usually somewhat lower than expected from the weighing $(\approx 1 \%)$, a difference which was assumed to originate from adsorbed water in the solid phase.

Acidified protocatechuic acid (3,4-dihydroxybenzoic acid, Sigma) solutions were prepared and analyzed in the same way as the gallic acid and the pyrogallol solutions. The titrated amounts of $\mathrm{C}_{7} \mathrm{H}_{6} \mathrm{O}_{4}$ were also lower $(\approx 0.5 \%)$ than that expected from weighing.

Special precautions. Aqueous solutions of almost all $o$-dihydroxyaryl compounds are susceptible to oxidation. The kinetics of this oxidation is, however, strongly retarded by the addition of strong acid, and this made it possible to prepare stock solutions of the three compounds. To avoid effects from the slow oxidation which also occurs in these solutions, the solutions were kept in the dark and used within a few days after standardization. The titrations were performed in airtight vessels with a gas outlet beneath a liquid surface, and before the addition of $\mathrm{OH}^{-}$, the vessel was deaerated with argon or hydrogen gas for at least $2 \mathrm{~h}$. This implies that all measurements were performed under strictly oxygen-free conditions, i.e. no oxidation of the ligands occurred during the measurements and fully stable EMF recordings (to within $\pm 0.02 \mathrm{mV}$ ) were obtained. The gas was passed through solutions of $10 \% \mathrm{NaOH}, 10 \%$ $\mathrm{H}_{2} \mathrm{SO}_{4}$, alkaline pyrogallol and a pure ionic medium before it reached the titration vessel. With these precautions, the protocatechuic acid and the gallic acid solutions could be titrated with $\mathrm{NaOH}$ solutions without visible discolouration. With the pyrogallol solutions it was, however, found that the minute amounts of oxygen dissolved in the $\mathrm{NaOH}$ solution also had to be avoided. This system has therefore been investigated through the use of coulometrically generated hydroxide ions.

The preparation of point solutions for the NMR measurements was performed in an argon-filled glove bag $\left(I^{2} R\right.$ instruments).

\section{Methods}

Potentiometric measurements. The measurements were performed at $25.00 \pm 0.05^{\circ} \mathrm{C}$ in an ionic medium consisting of $0.6 \mathrm{M} \mathrm{Na}(\mathrm{Cl})$. In the measurements, glass electrodes (Ingold $201 \mathrm{NS}$ ) and/or hydrogen electrodes were used. The potentiometric titrations were performed using an automatic system ${ }^{4}$ and $\mathrm{OH}^{-}$was added either using a $\mathrm{NaOH}$ solution or coulometrically. The measured EMF of the electrode, $E$, is given by eqn. (1), in which $E_{0}$ is a constant

$E=E_{0}+59.156 \log h+j_{\mathrm{ac}} h+j_{\mathrm{alk}} k_{\mathrm{w}} h^{-1}$

which is determined within each titration in solutions of known $\mathrm{H}^{+}$concentration, $h$ is the free $\mathrm{H}^{+}$concentration, $k_{\mathrm{w}}$ is the ionic product of water in $0.6 \mathrm{M} \mathrm{Na}(\mathrm{Cl})$ medium and $j_{\mathrm{ac}}$ and $j_{\mathrm{alk}}$ are coefficients in the expression for the liquid junction potential $\left[\log k_{\mathrm{w}}=-13.727, j_{\mathrm{ac}}=-77.1 \mathrm{mV} \mathrm{M}^{-1}\right.$ and $j_{\text {alk }}=42.0 \mathrm{mV} \mathrm{M}^{-1}$ in $\left.0.6 \mathrm{M} \mathrm{Na}(\mathrm{Cl})\right]^{5}$

The reproducibility and reversibility of equilibria were tested by performing both forward (increasing $-\log h$ ) and backward (decreasing $-\log h$ ) titrations. To avoid oxygen contamination in the second case, the $\mathrm{H}^{+}$-containing burette was mounted on the vessel from the beginning of the experiment.

The dissociation constants for the three compounds were separately determined from titrations in the absence of silicic acid. In all three systems these constants were found to be independent of the total ligand concentration. This validates the assumption that their possible contributions to the liquid junction potential are of negligible importance.

The three-component titrations were performed at a constant ratio between the total concentration of silicic acic, $B$, and ligand, $C$. These measurements were characterized by fast attainment of equilibrium at $-\log h \leqq 6$ and by equilibration times of ca. $4 \mathrm{~h}$ above this limit. 
Table 1. Mono- and dinuclear hydrolysis products of silicic acid. ${ }^{a}$

\begin{tabular}{rlcl}
\hline$p, q, r$ & Proposed formula & $\log \beta_{p, q, r}$ & Ref. \\
\hline-110 & $\mathrm{SiO}(\mathrm{OH})_{3}{ }^{-}$ & -9.473 & 5 \\
-210 & $\mathrm{SiO}_{2}(\mathrm{OH})_{2}{ }^{2-}$ & -22.12 & 5 \\
020 & $\mathrm{Si}_{2} \mathrm{O}(\mathrm{OH})_{6}$ & 1.2 & 6 \\
-120 & $\mathrm{Si}_{2} \mathrm{O}_{2}(\mathrm{OH})_{5}{ }^{-}$ & -7.75 & 2 \\
-220 & $\mathrm{Si}_{2} \mathrm{O}_{3}(\mathrm{OH})_{4}{ }^{2-}$ & -18.00 & 2 \\
\hline
\end{tabular}

aThe formation constants $\beta_{p, q, r}$ are defined according to the reaction $p \mathrm{H}^{+}+q \mathrm{Si}(\mathrm{OH})_{4}+n \mathrm{H}_{n} \mathrm{~L} \rightleftarrows \mathrm{H}_{p}\left(\mathrm{Si}(\mathrm{OH})_{4}\right)_{q}\left(\mathrm{H}_{n} \mathrm{~L}\right)_{r}^{p}$ and the values given are valid in $0.6 \mathrm{M} \mathrm{Na}(\mathrm{Cl})$ medium at $25^{\circ} \mathrm{C}$.

${ }^{29} \mathrm{Si}$-NMR measurements. All NMR measurements were performed at $295 \pm 1 \mathrm{~K}$ using a Bruker AC-250 spectrometer equipped with a $10 \mathrm{~mm}$ multinuclear probehead. During the measurements an inner concentric tube $(2 \mathrm{~mm}$ o.d.) with $\mathrm{D}_{2} \mathrm{O}$ was used as an instrumental lock. The chemical shifts were recorded with respect to $\mathrm{Si}\left(\mathrm{CH}_{3}\right)_{4}$ (tetramethylsilane, TMS) using the high-frequency-positive convention. The spectra (recorded with $16 \mathrm{~K}$ data) covered the region -70 to $-150 \mathrm{ppm}$, and the chemical shift zero was regularly checked by running TMS between each experimental solution. The spectra were recorded with 256 transients using $90^{\circ}$ pulses and pulse repetition times of $120 \mathrm{~s}$.

\section{Data treatment}

The equilibria to be considered in the present study can be divided into groups as follows.

(a) The binary $H^{+}$-ligand equilibria. These can be represented by the general reaction (2), in which $n=3$ for protocatechuic acid and pyrogallol and $n=4$ for gallic acid.

$$
\mathrm{H}_{n} \mathrm{~L} \rightleftarrows \mathrm{H}_{n-p} \mathrm{~L}^{-p}+p \mathrm{H}^{+} \quad \log \beta_{-p 01}
$$

In the present study, the carboxylic acid and the first hydroxylic dissociation constant have, for each ligand, been evaluated from separate titration experiments at $B=0$.

(b) The hydrolytic equilibria of $\mathrm{Si}(\mathrm{OH})_{4}$. The deprotonation of silicic acid in aqueous solution can, in dilute solutions, be described by reactions (3)-(7).

$$
\begin{array}{ll}
\mathrm{Si}(\mathrm{OH})_{4} \rightleftarrows \mathrm{SiO}(\mathrm{OH})_{3}{ }^{-}+\mathrm{H}^{+} & \log \beta_{-110} \\
\mathrm{Si}(\mathrm{OH})_{4} \rightleftarrows \mathrm{SiO}_{2}(\mathrm{OH})_{2}{ }^{2-}+2 \mathrm{H}^{+} & \log \beta_{-210} \\
2 \mathrm{Si}(\mathrm{OH})_{4} \rightleftarrows \mathrm{Si}_{2} \mathrm{O}(\mathrm{OH})_{6}+\mathrm{H}_{2} \mathrm{O} & \log \beta_{020} \\
2 \mathrm{Si}(\mathrm{OH})_{4} \rightleftarrows \mathrm{Si}_{2} \mathrm{O}_{2}(\mathrm{OH})_{5}{ }^{-}+\mathrm{H}^{+}+\mathrm{H}_{2} \mathrm{O} & \log \beta_{-120} \\
2 \mathrm{Si}(\mathrm{OH})_{4} \rightleftarrows \mathrm{Si}_{2} \mathrm{O}_{3}(\mathrm{OH})_{4}{ }^{2-}+2 \mathrm{H}^{+}+\mathrm{H}_{2} \mathrm{O} & \log \beta_{-220}
\end{array}
$$

For these equilibria, results evaluated in earlier parts of this series ${ }^{2,5,6}$ will be used. Equilibrium constants, valid in $0.6 \mathrm{M} \mathrm{Na}(\mathrm{Cl})$ medium, are given in Table 1.

(c) Three-component equilibria. The equilibria to be considered in the systems $\mathrm{H}^{+}-\mathrm{Si}(\mathrm{OH})_{4}-\mathrm{H}_{n} \mathrm{~L}$ are of the general form (8).

$p \mathrm{H}^{+}+q \mathrm{Si}(\mathrm{OH})_{4}+r \mathrm{H}_{n} \mathrm{~L} \rightleftarrows \mathrm{H}_{p}\left(\mathrm{Si}(\mathrm{OH})_{4}\right)_{q}\left(\mathrm{H}_{n} \mathrm{~L}\right)_{r}^{p+} \beta_{p q r}$

In the mathematical treatment of these data, the binary complex models under (a) and (b) were considered as known, and all effects above that level treated as being caused by the formation of three-component complexes.

Appplying the law of mass action and the condition for the total concentrations to these equilibria then gives eqns. (9)-(11), in which $b$ and $c$ are the free concentrations

$$
\begin{aligned}
& H=h-\Sigma p \beta_{-p 01} h^{-p} c-\Sigma p \beta_{-p q 0} h^{-p} b^{q}+\Sigma p \beta_{p q r} h^{p} b^{q} c^{r}-k_{w} h^{-1} \\
& B=b+\Sigma q \beta_{-p q 0} h^{-p} b^{q}+\Sigma q \beta_{p q r} h^{p} b^{q} c^{r} \\
& C=c+\Sigma \beta_{-p 01} h^{-p} c+\Sigma r \beta_{p q r} h^{p} b^{q} c^{r}
\end{aligned}
$$

of silicic acid and ligand, respectively. The summation is taken over all species formed, where $\beta_{-p 01}, \beta_{-p q 0}$ and $\beta_{p q r}$ are the equilibrium constants for reactions (2)-(8).

In the range of complex formation, $h$ was obtained for each point from the measured EMF using eqn. (1). Since $H$ and $C$ [eqns. (9) and (11)] are known from analysis, $Z_{c}$, defined as the average number of $\mathrm{OH}^{-}$reacted per $\mathrm{H}_{n} \mathrm{~L}$, can be calculated for each point in a titration according to eqn. (12).

$$
Z_{c}=\left(h-H-k_{w} h^{-1}\right) / C
$$

The computational problem now involves the determination of sets of pqr-triplets and corresponding equilibrium constants that 'best' fit the experimental data. In the evaluation of experimental data, standard graphical methods, as well as calculations using the least-squares computer program LETAGROPVRID $^{7}$ (version ETITR), ${ }^{7-9}$ were used. As 'best' model or models, those giving the lowest error-squares sum $U=\Sigma\left(H_{\text {calc }}-H_{\text {exp }}\right)^{2}$ were considered. The LETAGROP calculations also give standard deviations $\sigma(H)$ and $3 \sigma(\log \beta)$, calculated and defined according to Sillén. ${ }^{10,11}$

In order to visualize the fractions of the different species, the computer program SOLGASWATER,,$^{12}$ equipped with plotting procedures, was used.

The computations were performed on a CD Cyber 850 computer.

\section{Data, calculations and results}

The $H^{+}-H_{\mathrm{n}} L$ systems. Deprotonation constants of the different acids were determined from data within the concen- 
Table 2. First (and second) deprotonation constants of the different acids. ${ }^{a}$

\begin{tabular}{|c|c|c|c|c|c|}
\hline \multirow[t]{2}{*}{$p, q, r$} & \multirow[t]{2}{*}{ Proposed formula } & \multicolumn{4}{|c|}{$\log \left(\beta_{-n, 0,1} \pm 3 \sigma\right)$} \\
\hline & & Gallic acid & Protocatechuic acid & Pyrogallol & Pyrocatechol $^{b}$ \\
\hline$-1,0,1$ & $\mathrm{H}_{n-1} \mathrm{~L}^{-}$ & $\begin{array}{l}-4.141 \\
\pm 0.004\end{array}$ & $\begin{array}{l}-4.222 \\
\pm 0.007\end{array}$ & $\begin{array}{l}-8.905 \\
\pm 0.008\end{array}$ & $\begin{array}{l}-9.198 \\
\pm 0.001\end{array}$ \\
\hline$-2,0,1$ & $\mathrm{H}_{n-2} \mathrm{~L}^{2-}$ & $\begin{array}{r}-12.55 \\
\pm 0.012\end{array}$ & $\begin{array}{l}-12.87 \\
\quad \pm 0.021\end{array}$ & - & $\begin{array}{l}- \\
-\end{array}$ \\
\hline \multicolumn{2}{|l|}{$\sigma(H) / \mathrm{mM}$} & 0.07 & 0.07 & 0.05 & \\
\hline \multicolumn{2}{|c|}{ No. of titrations / no. of data } & $10 / 228$ & $5 / 122$ & $14 / 327$ & \\
\hline
\end{tabular}

${ }^{a}$ The equilibrium constants $\beta_{p, q, r}$ are defined in Table 1 and the errors given are $3 \sigma\left(\log \beta_{p, q, r}\right) .{ }^{b}$ From Ref. 1.

tration ranges $0.004 \leq C \leq 0.03 \mathrm{M}$ and $2.1 \leqq-\log h \leqq 9.5$. Results of the different LETAGROP calculations are presented in Table 2. In the gallic acid system, the carboxyl acidity constant $\mathrm{p} K_{\mathrm{a}}(\mathrm{COOH})=4.141$ was obtained. Furthermore, the corresponding value for the most acidic $\mathrm{OH}$ group, $\mathrm{p} K_{\mathrm{a}}(\mathrm{OH})=8.41$, was found. These two values are in good agreement with those previously reported by Öhman and Sjöberg ${ }^{13}$ (4.152 and 8.43, respectively). In the protocatechuic acid system, $\mathrm{p} K_{\mathrm{a}}(\mathrm{COOH})=4.22$ and $\mathrm{p} K_{\mathrm{a}}(\mathrm{OH})=8.65$, while $\mathrm{p} K_{\mathrm{a}}(\mathrm{OH})=8.90$ was found in the pyrogallol system. The differences in these values, reflecting the substitution effects on the pyrocatechol molecule, will be discussed in more detail below.

No attempts to determine $\mathrm{p} K_{\mathrm{a}}$-values for the other hydroxyl groups of the ligands were performed. The reasons for this are that in such alkaline solutions, the ligands very easily become oxidized. Furthermore, reliable

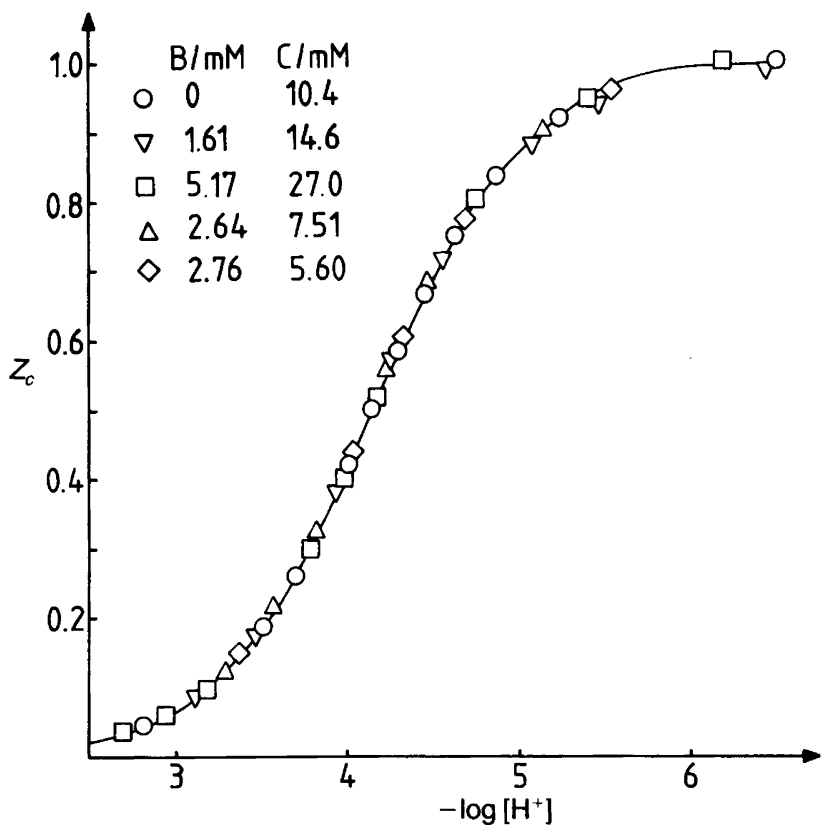

Fig. 1. Part of experimental data in the $\mathrm{H}^{+}-\mathrm{Si}(\mathrm{OH})_{4}$-gallic acid system plotted as curves $Z_{c}\left(-\log \left[\mathrm{H}^{+}\right]\right)$for $Z_{c} \leq 1$. The full curve was calculated with $\log \beta_{-1,0,1}=-4.141$. glass electrode data are difficult to obtain at the high $-\log h$-values needed for this determination.

The $\mathrm{H}^{+}-\mathrm{Si}(\mathrm{OH})_{4}-\mathrm{H}_{\mathrm{n}} L$ systems. During the three-component titrations, the ratio between $B$ and $C$ was held constant. The initial concentrations were varied within the limits $0.001 \leq B \leq 0.006 \mathrm{M}$ and $0.002 \leq C \leq 0.03 \mathrm{M}$, covering the $C / B$ ratios $1 \leq C / B \leq 15$. The measurements of $\left[\mathrm{H}^{+}\right]$were performed within the range $2.5 \leqq-\log h \leqq 9.5$.

The mathematical treatment of the data was started by calculating $Z_{c}(-\log h)$ curves. In the gallic acid system (cf. Fig. 1) it can be noted that no interactions with $\mathrm{Si}(\mathrm{OH})_{4}$ are found with $-\log h \leqq 6$ or $Z_{c} \leq 1$. However, in neutral and slightly alkaline solutions, the increased buffer capacity of the system (Fig. 2) clearly indicates complex formation with silicic acid.

To determine the composition and stability of the threecomponent complex(es) that are formed, a systematic testing of different complexes was performed. The results of the calculations in the $\mathrm{H}^{+}-\mathrm{Si}(\mathrm{OH})_{4}$-gallic acid system are visualized in Fig. 3. As can be seen, the best fit was obtained by the $p, q, r$ combination $-5,1,3$. This combination also yielded the best fit for the protocatechuic acid system,

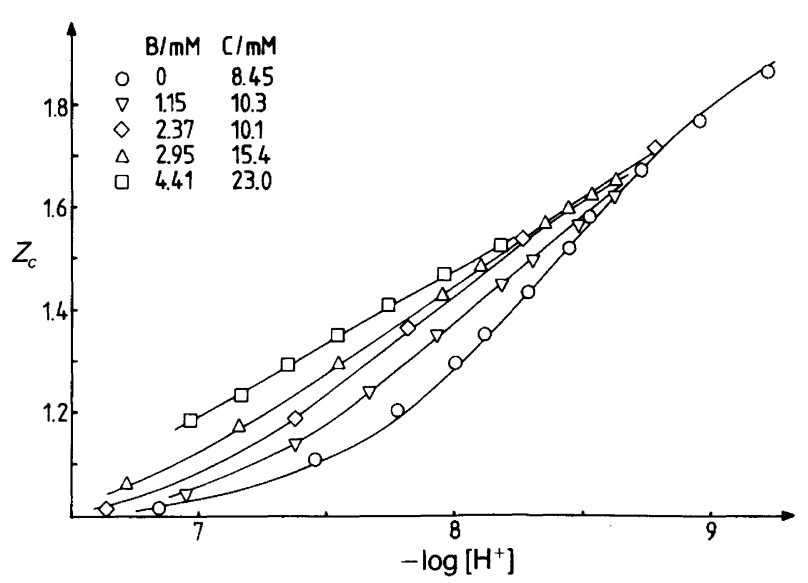

Fig. 2. Some of the experimental data at $Z_{c} \geq 1$ for the $\mathrm{H}^{+}-\mathrm{Si}(\mathrm{OH})_{4}$-gallic acid system. All symbols represent initial concentratioans at $Z_{c}=1$ and the curves were constructed using the proposed equilibrium model. 

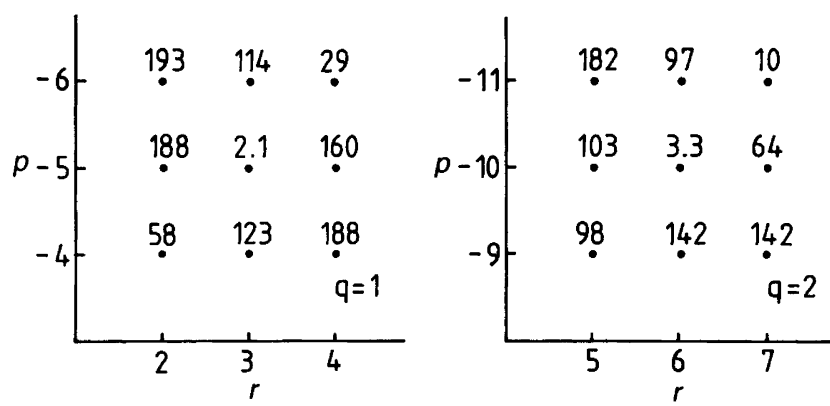

Fig. 3. Result of $p, q, r$ analysis on data with $Z_{c} \geq 1$ in the system $\mathrm{H}^{+}-\mathrm{Si}(\mathrm{OH})_{4}$-gallic acid. The figures give error-squares sums $U_{\mathrm{H}}(p r)_{q}$ assuming the formation of one ternary complex.

while for the pyrogallol system the corresponding 'best' combination was $-2,1,3$ (not illustrated). These results clearly show the formation of a complex with a ligand-to-Si ratio of 3 and with a charge of -5 in the gallic acid and protocatechuic acid systems, and of -2 with pyrogallol. Although the remaining residuals, $H_{\text {calc }}-H_{\text {exp }}$, showed no systematic trend, speciation models where series of complexes $-5 /-2,1, r,(r=1,2,3)$ and $0,1, r,(r=1,2)$ together with $-5 /-2,1,3$ were also tested. However, in neither of these cases was any significant improvement of the fit obtained. Although small amounts of a disilicic acid are present at the highest $B$-values studied, no polynuclear complexes with the different ligands could be established.

It may thus be concluded that equilibria (13)-(15) have been determined. The corresponding formation constants are given in Table 3.

$$
\begin{aligned}
& \mathrm{Si}(\mathrm{OH})_{4}+3 \mathrm{H}_{3} \mathrm{~L} \rightleftarrows \mathrm{Si}(\mathrm{HL})_{3}{ }^{2-}+2 \mathrm{H}^{+}+4 \mathrm{H}_{2} \mathrm{O} ; \\
& \text { (pyrogallol) } \\
& \mathrm{Si}(\mathrm{OH})_{4}+3 \mathrm{H}_{3} \mathrm{~L} \rightleftarrows \mathrm{SiL}_{3}^{5-}+5 \mathrm{H}^{+}+4 \mathrm{H}_{2} \mathrm{O} ; \\
& \text { (protocatechuic acid) } \\
& \mathrm{Si}(\mathrm{OH})_{4}+3 \mathrm{H}_{4} \mathrm{~L} \rightleftarrows \mathrm{Si}(\mathrm{HL})_{3}{ }^{5-}+5 \mathrm{H}^{+}+4 \mathrm{H}_{2} \mathrm{O} \\
& \text { (gallic acid) }
\end{aligned}
$$
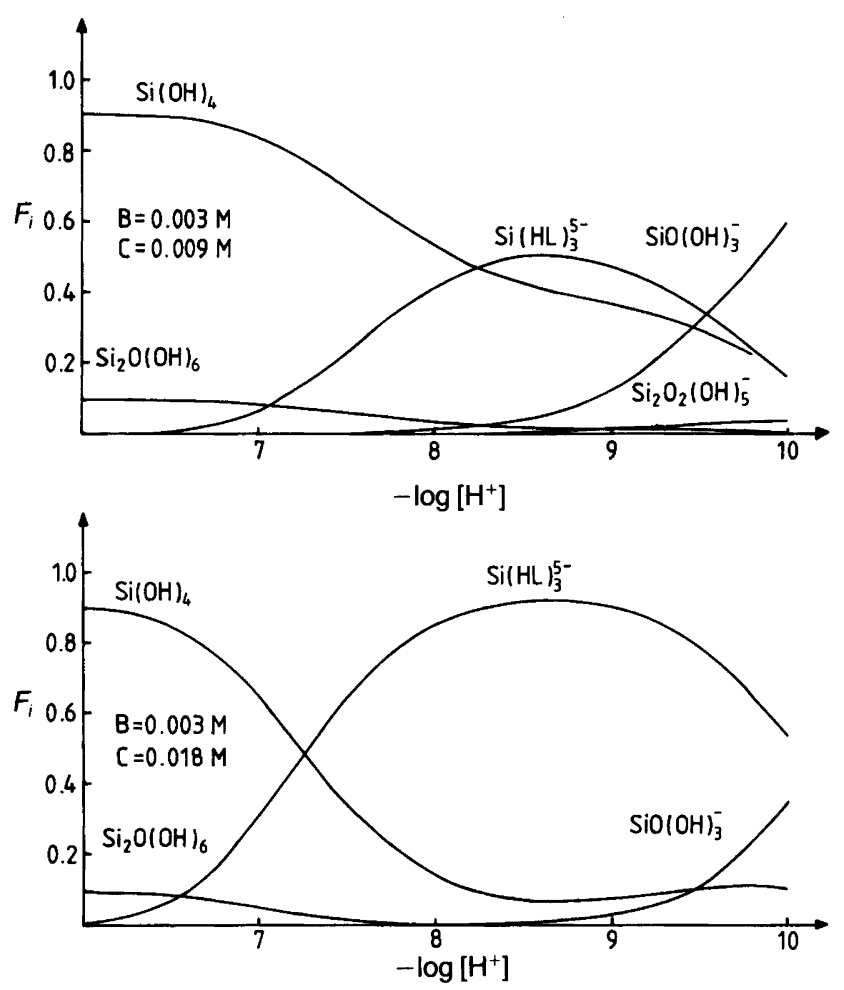

Fig. 4. Distribution diagrams $F_{i}\left(-\log \left[\mathrm{H}^{+}\right]\right)$in the

$\mathrm{H}^{+}-\mathrm{Si}(\mathrm{OH})_{4}-$ gallic acid system. $F_{i}$ is defined as the ratio between $\mathrm{Si}$ in a species and total $\mathrm{Si}$ concentration.

In order to visualize the conditions under which $\mathrm{SiL}_{3}{ }^{5-}$ is formed in the gallic acid system, the computer program SOLGASWATER was used to calculate some distribution diagrams. These are presented in Fig. 4 and show that $\mathrm{Si}(\mathrm{HL})_{3}{ }^{5-}$ is formed to a maximum amount in the $-\log h$ range 8.5-9. Also, with the other two ligands (not illustrated) the highest fraction of $\mathrm{SiL}_{3}$ was obtained at $-\log h$ $\approx 9$. This was therefore the $-\log h$ value chosen at the preparation of point solutions for ${ }^{29} \mathrm{Si}-\mathrm{NMR}$ characterization. In addition, to avoid problems with precipitation of

\begin{tabular}{|c|c|c|c|c|c|}
\hline \multirow[t]{2}{*}{$p, q, r$} & \multirow[t]{2}{*}{ Proposed formula } & \multicolumn{4}{|c|}{$\log \left(\beta_{p, q, r} \pm 3 \sigma\right)$} \\
\hline & & Gallic acid & Protocatechuic acid & Pyrogallol & Pyrocatechol $^{b}$ \\
\hline$-5,1,3$ & $\mathrm{Sil}_{3}{ }^{5-}$ & $\begin{array}{c}-21.26 \\
\pm 0.03\end{array}$ & $\begin{array}{r}-21.95 \\
\pm 0.05\end{array}$ & - & $\begin{array}{l}- \\
-\end{array}$ \\
\hline$-2,1,3$ & $\mathrm{Sil}_{3}^{2-}$ & - & - & $\begin{array}{r}-10.02 \\
\pm 0.01\end{array}$ & \multirow[t]{2}{*}{$\begin{array}{c}-10.44 \\
\pm 0.03\end{array}$} \\
\hline$\sigma(H) / \mathrm{mM}$ & & 0.08 & 0.08 & 0.08 & \\
\hline \multicolumn{2}{|c|}{ No. of titrations / no. of data } & $15 / 301$ & $11 / 212$ & $9 / 218$ & \\
\hline
\end{tabular}
amorphous silica, these solutions were prepared using an excess of ligand $(B=0.01 \mathrm{M} ; C=0.05 \mathrm{M})$.

In all three spectra, one dominating resonance was observed: at $-142.8 \mathrm{ppm}$ vs. TMS for pyrogallol, at -142.4

Table 3. Stability constants for the different $\mathrm{SiL}_{3}$ complexes. $^{a}$

${ }^{a}$ The constants $\beta_{p, q, r}$ are defined in Table 1 and the errors given are $3 \sigma\left(\log \beta_{p, q, r}\right) .{ }^{b}$ From Ref. 1. 
FATTAHPOUR SEDEH ET AL.

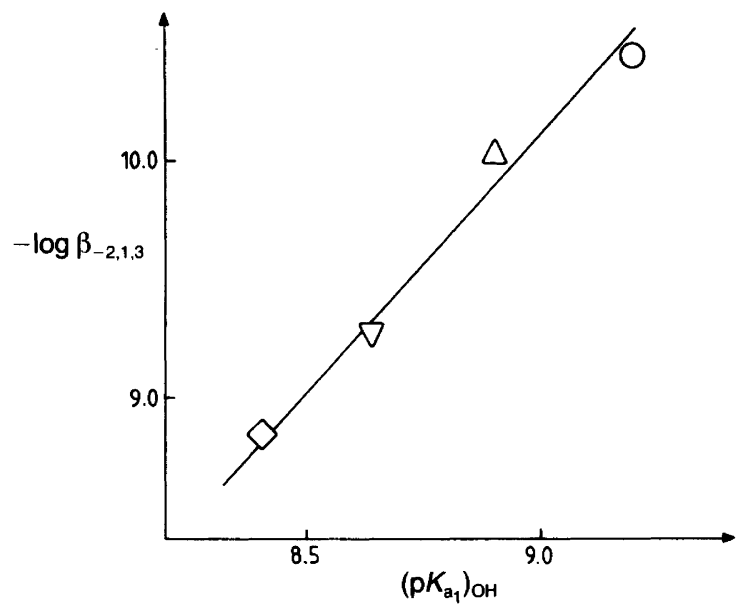

Fig. 5. Logarithm of the stability constant for $\mathrm{SiL}_{3}$ written as $\log \beta_{-2,1,3}\left[\mathrm{Si}(\mathrm{OH})_{4}+3 \mathrm{H}_{n} \mathrm{~L}^{-x} \rightleftarrows \mathrm{Si}\left(\mathrm{H}_{n-2} \mathrm{~L}\right)_{3}^{-(2+3 x)}+2 \mathrm{H}^{+}+4 \mathrm{H}_{2} \mathrm{O}\right.$; $x=0$ or 1] versus the first phenolic $\mathrm{p} K_{\mathrm{a}}$ of the ligand. From left to right the symbols represent gallic acid, protocatechuic acid, pyrogallol and pyrocatechol.

ppm for protocatechuic acid and at $-140.9 \mathrm{ppm}$ for gallic acid. Since chemical shift values in this range are typical for a silicon atom surrounded with six oxygens in an octahedral configuration, ${ }^{14}$ these spectra thus provided a strong indication of the formation of hexacoordinated complexes with bidentately bound ligands.

\section{Discussion}

Speciation and equilibria. The present study has confirmed that silicic acid forms a stable hexacoordinated tris-complex with $o$-diphenolic groups in slightly alkaline aqueous solutions. It has also been shown that the charge and the stability of the resulting species are a function of the substituent pattern on the pyrocatechol molecule.

From previous empirical findings it is known that, for a series of structurally related ligands, a plot of the logarithm of the stability constant versus a parameter representing the inductive effect of the substituent usually gives a straight line. This technique has been widely used in organic chemistry, having been used in 1934 by Larsson ${ }^{15}$ with regard to complex formation. The technique was later applied $^{16}$ to correlate the complexing ability of related ligands or metal ions.

Using a ligand with acidic or basic properties, the best correlations have usually been obtained by plotting $\log K$ for complex formation vs. $\mathrm{p} K_{\mathrm{a}}$ of the ligand. With the present ligands it is, however, important to realize that for such a correlation to be valid, all equilibria must emerge from a comparable reference state. Since the carboxylic acid groups of gallic acid and protocatechuic acid are only indirectly involved in complexation, this implies that the reactions of pyrogallol and pyrocatechol ${ }^{1}$ should be compared to those of the gallate(-1) and protocatechuate(-1) ions. The equilibrium constants to be correlated are thus $\log \beta_{-2,1,3}\left[\mathrm{Si}(\mathrm{OH})_{4}+3 \mathrm{H}_{n} \mathrm{~L}^{x-} \rightleftarrows \mathrm{Si}\left(\mathrm{H}_{n-2} \mathrm{~L}\right)_{3}{ }^{-(2+3 x)}+2 \mathrm{H}^{+}+\right.$ $4 \mathrm{H}_{2} \mathrm{O}, x=0$ or 1] against the first phenolic $\mathrm{p} K_{\mathrm{a}}$ of the

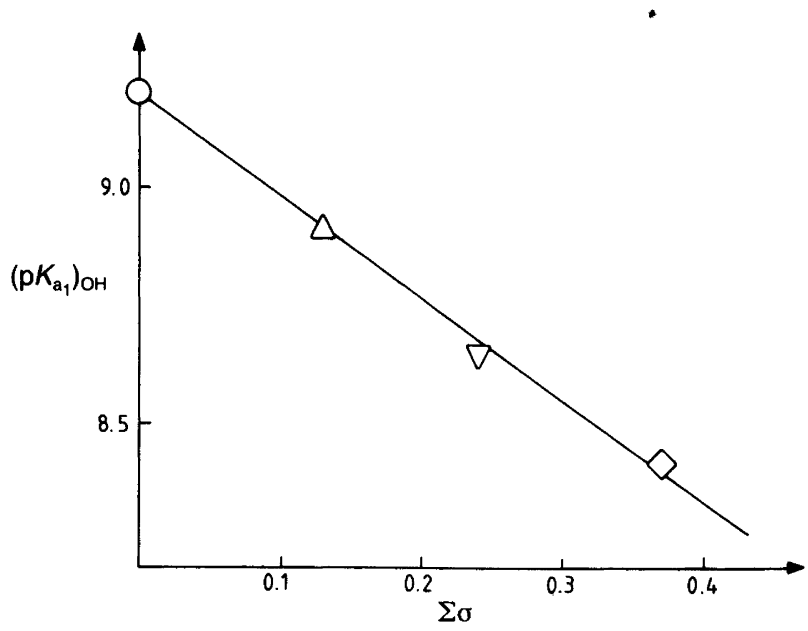

Fig. 6. The first phenolic $p K_{\mathrm{a}}$ of, from left to right, pyrocatechol, pyrogallol, protocatechuic acid and gallic acid versus the sum of Hammett substituent parameters with pyrocatechol as parent compound.

ligand. This plot is presented in Fig. 5, and the data correlate well with a straight line.

Acceptable linear correlations can also be obtained by using an empirical parameter, $\sigma$, suggested by Hammett, ${ }^{17}$ instead of $\mathrm{p} K_{\mathrm{a}}$. This parameter is introduced through the Hammett equation, eqn.(16), which relates the equilibrium

$\mathrm{p} K=\mathrm{p} K^{\circ}-\mathrm{\varrho} \Sigma \sigma$

constant $K$ relative to the constant $K^{\circ}$ for a parent compound to a parameter $\varrho$, characteristic of the specific reaction, and $\sigma$, the substituent parameter.

According to eqn. (16), combined with the additivity principle, a pyrocatechol molecule substituted as in Scheme 1 should therefore have predictable acid-base and complexation properties as long as the 'external-ring' inter-

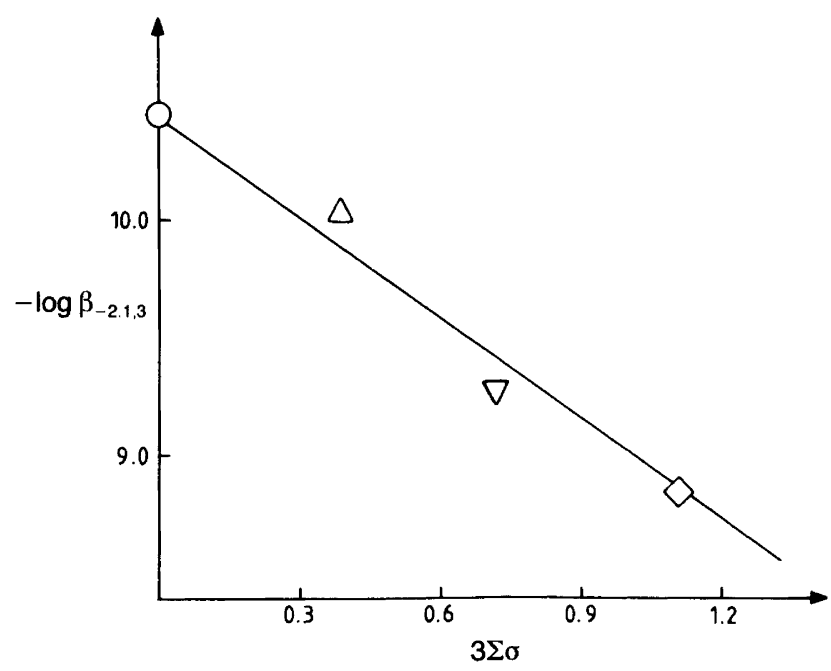

Fig. 7. Logarithm of $\log \beta_{-2,1,3}$ (cf. Fig. 5) plotted versus three times the sum of Hammett substituent parameters. The symbols used are the same as in Fig. 6. 


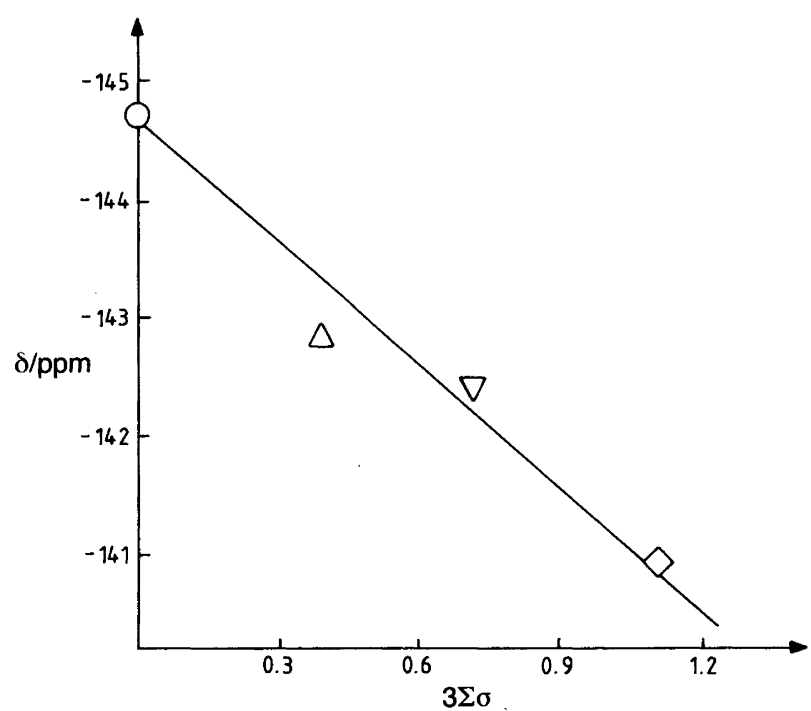

Fig. 8. Chemical shift of ${ }^{29} \mathrm{SiL}_{3}$ (in ppm vs. TMS) plotted against three times the sum of Hammett substituent parameters. The symbols used are the same as in Fig. 6 and the value for pyrocatechol (-144.7 ppm) was taken from Ref. 1.

actions between $\mathrm{Y}$ and the adjacent $\mathrm{OH}$ group are negligible. By using substituent parameters from the literature $^{17.18}$ and pyrocatechol as the parent compound, the sum of substituent parameters for the present compounds becomes 0.13 for pyrogallol, 0.24 for the protocatechuate $(-1)$ ion and 0.37 for the gallate(-1) ion. In Fig. 6 these sums have been plotted against the first $\mathrm{p} K_{\mathrm{a}}$-value of the compounds and, as seen, a good linear correlation is obtained. Furthermore, the slope of the resulting line, $\varrho=2.21$, shows good agreement with the slope reported in the literature $^{19}$ for phenolic compounds, $\varrho=2.23$.

As indicated in Fig. 5, the different substitutions also affect the stability of the three-component complex $\mathrm{SiL}_{3}$. Since $\log \beta_{-2.1 .3}$ and $\left(\mathrm{p} K_{\mathrm{a} 1}\right)_{\mathrm{OH}}$ showed a linear correlation (Fig. 5) and (pK $\left.K_{\mathrm{a}_{1}}\right)_{\mathrm{OH}}$ is linearly correlated to $\Sigma \sigma$ (Fig. 6), a linear correlation is also to be expected between $\log \beta_{-2,1.3}$ and the sum of substituent parameters. This plot, in which $\Sigma \sigma$ has been multiplied by three to take into account the three ligands in the complex, is given in Fig. 7, and, as foreseen, an approximate straight line is obtained. The slope of this line, $\varrho=1.4$, is lower than the corresponding slope for the $\left(\mathrm{p} K_{\mathrm{a}_{1}}\right)_{\mathrm{OH}}$ line. This implies that a net strengthening of $\mathrm{SiL}_{3}$ is obtained with increasing values of $\Sigma \sigma$.

As was noted from the NMR experiments, all $\mathrm{SiL}_{3}$ complexes give a single resonance at ca. -141 to $-145 \mathrm{ppm}$ vs. TMS; values which were taken as evidence for octahedrally coordinated silicon complexes. In Fig. 8 these chemical shift values have been plotted against (three times) the sum of substituent parameters. The linearity observed in this plot indicates that the Hammett equation might also be used for correlating chemical shift data from structurally related compounds.

Modelling calculations. Owing to the high abundance of silicon in the crustal rock, silicon compounds are a major

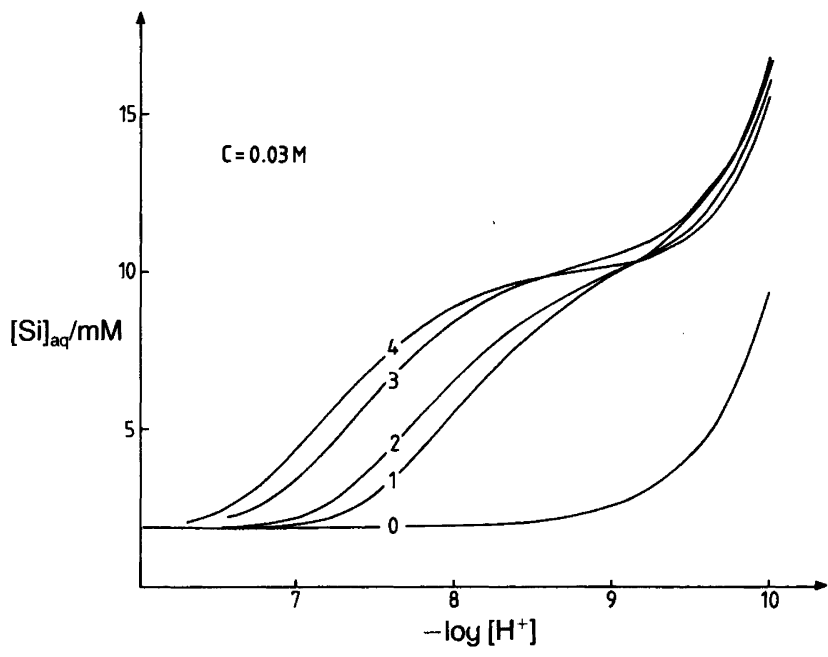

Fig. 9. Theoretical solubility of amorphous $\mathrm{SiO}_{2}\left(\log K_{\text {so }}=\right.$ -2.76 ) in absence $(0)$ and presence of $0.03 \mathrm{M}$ pyrocatechol (1), pyrogallol (2), protocatechuic acid (3) or gallic acid (4) as a function of $-\log \left[\mathrm{H}^{+}\right]$.

component of the suspended matter derived from weathering processes. Because of the low solubility of secondary solid phases formed, such as aluminosilicates and different forms of silica, the aqueous silicon concentration does, however, seldom exceed the value predicted by the solubility of amorphous silica. Until recently, the prevailing forms of this silicon have commonly been referred to as the uncharged silicic acid, $\mathrm{Si}(\mathrm{OH})_{4}$, and, to a small extent, the dimer of this species, $\mathrm{Si}_{2} \mathrm{O}(\mathrm{OH})_{6}$.

This simple view has recently been challenged by work performed by Bennett et al. ${ }^{20-22}$ showing that the solubility of quartz is influenced by the presence of certain natural and synthetic organic compounds at near-neutral $\mathrm{pH}$. To indicate the potential influence of such reactions from the presently studied compounds, different model calculations have been performed.

In one calculation, amorphous silica $\left(\log K_{\mathrm{so}}=-2.76\right)^{1}$ was equilibrated with $3 \times 10^{-2} \mathrm{M}$ of ligand and the total

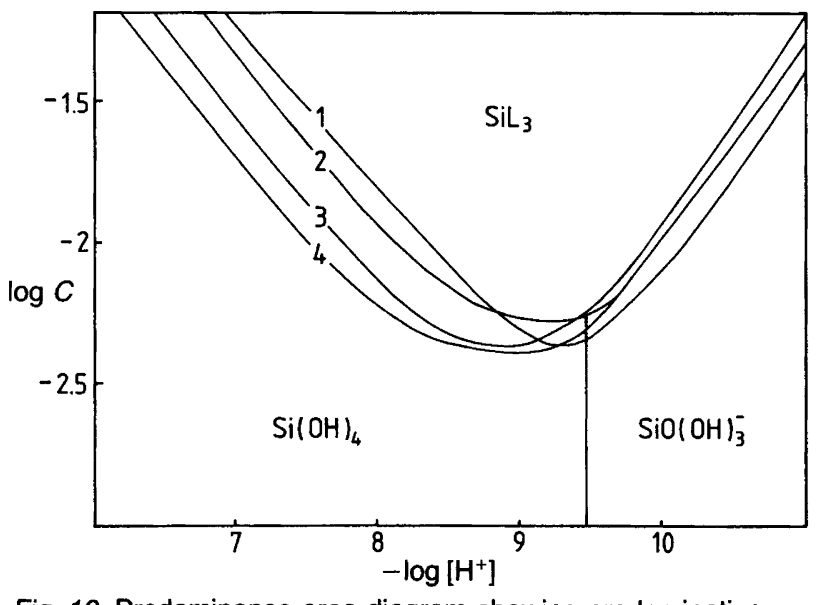

Fig. 10. Predominance area diagram showing predominating aqueous form of silicon in a suspension of quartz $\left(\log K_{\mathrm{so}}=-4\right)$ containing various total concentrations of pyrocatechol (1), pyrogallol (2), protocatechuic acid (3) or gallic acid (4). 


\section{FATTAHPOUR SEDEH ET AL}

aqueous silicon concentration was calculated as a function of $-\log h$. The resulting solubilization curves are illustrated in Fig. 9 and show, in accordance with the arguments given above, that the strongest solubilization effect is obtained with gallic acid. Thus, with this compound, silicon can be mobilized from solid silica even below neutral $-\log h$. The high solubility of amorphous silica and the 1:3 stoichiometry of the soluble species did, however, make it necessary to perform these calculations with relatively high ligand concentrations.

Another calculation was therefore performed in which crystalline quartz $\left(\log K_{\mathrm{so}}=-4\right)$ was used as a solubilityregulating phase. In Fig. 10 this calculation is illustrated in the form of a predominance area diagram, i.e. a diagram showing the predominating aqueous form of silicon in a solution in contact with crystalline quartz. This implies that the solubility of quartz has increased by a factor of two along the different $\mathrm{Si}(\mathrm{OH})_{4} / \mathrm{SiO}(\mathrm{OH})_{3}{ }^{-}-\mathrm{SiL}_{3}$ boundary lines. Once again, these calculations show that the strongest complexation effects are obtained with gallic acid as ligand.

Also here, however, such high ligand concentrations are needed that it is doubtful whether complexes of this type are of significance under 'normal' natural water conditions, for instance in streams or lake waters. Under more 'special' conditions on the other hand, e.g. the area investigated by Bennett and Siegel, ${ }^{20}$ where high concentrations of biodegraded petroleum were present in the water body, silicon complexes of this kind are quite likely to appear.

Acknowledgements. We are grateful to Dr. Michael Sjöström for useful discussions concerning the application of the Hammett equation. This work forms part of a program financially supported by the Swedish Natural Science Research Council.

\section{References}

1. Öhman, L.-O., Nordin, A., Fattahpour Sedeh, I. and Sjöberg, S. Acta Chem. Scand. 45 (1991) 335.

2. Sjöberg, S., Öhman, L.-O. and Ingri, N. Acta Chem. Scand., Ser. A 39 (1985) 93.

3. Gran, G. Acta Chem. Scand. 4 (1950) 559.

4. Ginstrup, O. Chem. Instrum. 4 (1973) 141.

5. Sjöberg, S., Nordin, A. and Ingri, N. Mar. Chem. 10 (1981) 521.

6. Sjöberg, S., Ingri, N., Nenner, A.-M. and Öhman, L.-O. J. Inorg. Biochem. 24 (1985) 267.

7. Ingri, N. and Sillén, L. G. Ark. Kemi 23 (1964) 97.

8. Arnek, R., Sillén, L. G. and Wahlberg, O. Ark. Kemi 31 (1969) 353.

9. Brauner, P., Sillén, L. G. and Whiteker, R. Ark. Kemi 31 (1969) 365.

10. Sillén, L. G. Acta Chem. Scand. 16 (1962) 159.

11. Sillén, L. G. and Warnqvist, B. Ark. Kemi 31 (1969) 341.

12. Eriksson, G. Anal. Chim. Acta 112 (1979) 375.

13. Öhman, L.-O. and Sjöberg, S. Acta Chem. Scand., Ser. A 35 (1981) 201.

14. Coleman, B. In: Laszlo, P., Ed., NMR of Newly Accessible Nuclei, Academic Press, New York 1983, Vol. 2, p. 211.

15. Larsson, E. Z. Phys. Chem. A 169 (1934) 207.

16. Irving, H. and Rosotti, H. Acta Chem. Scand. 10 (1956) 72.

17. Hammett, L. P. Physical Organic Chemistry, 2nd. ed., McGraw-Hill, New York 1970, p. 353.

18. Cohen, L. A. and Jones, W. M. J. Am. Chem. Soc. 85 (1963) 3397

19. Biggs, A. I. and Robinson, R. A. J. Chem. Soc. (1961) 388.

20. Bennett, P. C. and Siegel, D. T. Nature (London) 326 (1987) 684.

21. Bennett, P. C., Melcer, M. E., Siegel, D. I. and Hassett, J. P. Geochim. Cosmochim. Acta 52 (1988) 1521.

22. Bennett, P. C. Geochim. Cosmochim. Acta 55 (1991) 1781.

Received December 27, 1991. 